\title{
Pharmaceutical Care Services: Indian Scenario
}

Jagadevappa Patil*

VT's Shivajirao S Jondhale College of Pharmacy, Asangaon-421 601, Shahapur, Thane, Maharashtra, India

*Corresponding author: Jagadevappa Patil, VT's Shivajirao S Jondhale College of Pharmacy, Asangaon-412 601, Thane, Maharashtra, India, Tel: +91 9448816812; Email: pharmajspatil@gmail.com

Received date: May 24, 2017; Accepted date: May 25, 2017; Published date: May 31, 2017

Copyright: (c 2017 Patil J. This is an open-access article distributed under the terms of the Creative Commons Attribution License, which permits unrestricted use, distribution, and reproduction in any medium, provided the original author and source are credited.

Citation: Patil J (2017) Pharmaceutical Care Services: Indian Scenario. J Pharmacovigil 5: e169. doi:10.4172/2329-6887.1000e169

\section{Introduction}

The qualified pharmacist in coordination with other health care team works to prevent disease and to assess, monitor, initiate and modify use of medication in order to ensure safe and effective drug therapy regimens. This system is recognized as a patient focused, outcome based pharmaceutical care or pharmacy practice. This system is mainly aimed to optimize the patient health and to achieve positive clinical outcomes through the active involvement of clinical pharmacists in health care organizations. The active involvement of clinical pharmacists in India in this regard is not up to the expected mark in contrary to trends in developed countries. In present day health care trend usage of multiple drugs in drug therapy is common practice and raised the complexity of drug interactions and similar problems. To address such situations and solve the probable problems, existence of pharmaceutical care system in Indian health care organizations is of urgent need.

Pharmacists are highly qualified, well positioned health care personnel to assist the quality care in health care area. The designing, implementing and monitoring the therapeutic plan for best possible specific outcomes is a responsibility of pharmacist in coordination with other health care team members. This pharmaceutical care system is a result of long term thorough research and evolved as a generalist practice which can be successfully applied in all kinds of health care settings such a community, hospital, long term care and clinics. The pharmacists involved in health care practice is not intended to replace the physician, nurse or any other health care team members rather a new patient care provider with in the health care system.1 With enormous increase in use of medications leading to substantial increase in risk of medications errors and other drug related problems. The pharmacists as professional health care providers take care of safe, appropriate and cost-effective use of medications.

As per the WHO recommendations 1992, every health care organizations including community and hospital pharmacy must know about good pharmacy practices and these guidelines highlight the provision of pharmaceutical care. The general practices of pharmacists includes following standardized methods:

1. Collecting and arranging specific information about patients;

2. Identifying the probable medication therapy problems;

3. Enlisting patient's health care needs;

4. Specifying pharmacotherapeutic goals;

5. Designing the drug therapy regimen;

6. Preparing a specific monitoring plan;

7. Developing a specific drug therapy regimen in coordination with patient and other health care team members;

8. Monitoring the effects of drug therapy regimens;
9. Redesigning and monitoring the drug therapy regimens and plans;

10. Recommending the physician in selecting the right drug.

Pharmacists play vital role in solving such health care problems presented by complex health care system. Several pharmaceutical care programmes have been established in many countries to enhance clinical outcomes and improve health care system. These programmes are successfully implemented by pharmacists. However, such effective programmes do not exist in the Indian scenario. It is growing to bring down the effective pharmaceutical care system in present Indian health care system. India is country with significant problems in use of medications due to nonexistence of clinical pharmacy services and this is again because of huge resistance on part of medical professionals to accept the role of pharmacists in clinical aspects as well the reluctance of pharmacists themselves towards assuming such clinical role and responsibilities. However, this discouraging scenario has started changing slowly in India and many hospitals have initiated clinical pharmacy and pharmaceutical care services with significant positive results [1].

\section{Identified resistances}

There are many reasons identified in implementing pharmaceutical care services in Indian health care system. Following are few:

1. Mentality of medical professionals in accepting pharmacist's role in clinical affairs;

2. Lack of time;

3. Lack of qualified personnel;

4. Lack of documentation system;

5. Reluctance of pharmacists themselves in such role and responsibilities.

\section{Conclusion}

The pharmacist has dual role as team member and as individual practitioner in a health care system to ensure the quality health care services to the public. It is the responsibility of a pharmacist to understand his changing role as a health care provider other than just dispensing the medicines in a hospital as pharmaceutical care is patient oriented rather than product or profit centered. It is a clinical outcome based service where pharmacist can act properly in optimizing the drug therapy to achieve safe and effective patient service.

\section{References}

1. Heppler CD, Stran LM (1990) Opportunities and responsibilities in pharmaceutical care. Am J Hosp Pharm 47: 533-543. 